\title{
ON THE INDECOMPOSABILITY OF TORSION-FREE ABELIAN GROUPS
}

\author{
J. W. ARMSTRONG
}

1. We consider the following question posed by E. Weinberg $[4$, §5.2]: Does there exist a torsion-free abelian group of cardinality greater than the continuum $(\boldsymbol{N})$ with the property that each pure subgroup is (directly) indecomposable? In §2 we answer this question negatively for a large class of groups which contains, most notably, the class of homogeneous groups. In $\$ 3$ we characterize, in terms of indecomposability, the pure and $p$-pure subgroups of the $p$-adic integers.

Throughout this note all groups are abelian. The notation follows the usage in [2].

2. Definition. A purely indecomposable torsion-free group is a group every pure subgroup of which is indecomposable.

We shall prove the

THEOREM. Let $G$ be a torsion-free group of cardinality $m$ with the property: In the type set of $G$ there exists a type $\tau$ maximal with respect to the property that $m$ elements of $G$ have this type. If $G$ is purely indecomposable, then $m \leqq \aleph$.

The proof of this theorem is accomplished in four steps, the first three of which are lemmas.

Lemma 1. Let $G$ be a purely indecomposable torsion-free group. Let $g \in G$ and put $\bar{G}=G /\{g\}_{*}$. Then, for each $g_{\alpha} \in G$ with $T\left(g_{\alpha}\right)=T(g)$, $T\left(g_{\alpha}\right)<T\left(\bar{g}_{\alpha}\right)$.

Proof. We use a result of Baer [2, Lemma 46.3] which states that if $H$ is torsion free and if $S$ is a pure subgroup of $H$ such that (i) $H / S$ has rank 1, (ii) the type of $H / S$ is $\tau$, and (ii) each element of $H$ not in $S$ has type $\tau$, then $S$ is a direct summand of $H$. To prove the lemma, let $S=\{g\}_{*}$ and $H=\left\{g_{\alpha}, g\right\}_{*}$. Since $G$ is purely indecomposable and $H$ is pure in $G$, there must exist an element $x \in H, x \in S$, such that $T(x)>T\left(g_{\alpha}\right)$. Then for some integers $k, m$ and $n, k x=m g_{\alpha}$ $+n g$, and $T\left(\bar{g}_{\alpha}\right)=T(\bar{x}) \geqq T(x)>T\left(g_{\alpha}\right){ }^{1}$

Lemma 2. Let $G$ be torsion free, $g \in G$, and $\bar{G}=G /\{g\}_{*}$. Suppose there is an independent subset $S$ consisting of $m>\boldsymbol{N}$ elements of $G$ all

Presented to the Society, January 24,1964 ; received by the editors November 6 , 1963.

1 The author is indebted to the referee for this simple proof which replaces the lengthy original one. 
having the same height and an infinite set $\pi$ of primes such that $h_{p}\left(g_{\alpha}\right)$ $<h_{p}\left(\bar{g}_{\alpha}\right)$ for each $g_{\alpha} \in S$ and $p \in \pi$. Then $S$ contains a subset $T$ of cardinality $m$ such that whenever $g_{\alpha}, g_{\beta} \in T, g_{\alpha} \neq g_{\beta}$, then $T\left(g_{\alpha}-g_{\beta}\right)>T\left(g_{\alpha}\right)$.

Proof. Fix $g_{\alpha} \in S$ and $p \in \pi$. Let $h_{p}\left(g_{\alpha}\right)=k \geqq 0$. Then $h_{p}\left(\bar{g}_{\alpha}\right) \geqq k+1$ and there is $r_{\alpha, p} \in Q$ with $p^{k+1} x=g_{\alpha}+r_{\alpha, p} g, x \in G$. Thus, with each $g_{\alpha}$ we associate a sequence $\left\langle r_{\alpha, p}\right\rangle_{p \in \pi}$ of rational numbers. Considering cardinalities, we conclude that there are $m$ elements of $S$, all of which are associated with the same sequence. Denote the set of these elements by $T$. Then, whenever $g_{\alpha}, g_{\beta} \in T, g_{\alpha} \neq g_{\beta}$,

$$
h_{p}\left(g_{\alpha}-g_{\beta}\right) \geqq \min \left\{h_{p}\left(g_{\alpha}+r_{\alpha, p} g\right), h_{p}\left(g_{\beta}+r_{\beta, p} g\right)\right\}>h_{p}\left(g_{\alpha}\right),
$$

for each $p \in \pi$. Hence $T\left(g_{\alpha}-g_{\beta}\right)>T\left(g_{\alpha}\right)$.

Lemma 3. Let $G$ be torsion-free, $g \in G$, and $\bar{G}=G /\{g\}_{*}$. Suppose there is an independent subset $S$ of $G$ of cardinality $m>\aleph$ and a prime $p$ such that $h_{p}\left(\bar{g}_{\alpha}\right)=\infty$ for every $g_{\alpha} \in S$. Then there is a subset $T$ of $S$ of cardinality $m$ such that whenever $g_{\alpha}, g_{\beta} \in T, g_{\alpha} \neq g_{\beta}$, then $h_{p}\left(g_{\alpha}-g_{\beta}\right)=\infty$.

Proof. Fix $g_{\alpha} \in S$. For each positive integer $n$ there is $r_{\alpha, n} \in Q$ with $h_{p}\left(g_{\alpha}+r_{\alpha, n} g\right) \geqq n$. As in Lemma 2 , associate with each $g_{\alpha} \in S$ the sequence $\left\langle r_{\alpha, n}\right\rangle$. Considering cardinalities, we conclude there are $m$ elements of $S$ which are associated with the same sequence. Denote this set of elements by $T$. Then, whenever $g_{\alpha}, g_{\beta} \in T, g_{\alpha} \neq g_{\beta}$,

$$
h_{p}\left(g_{\alpha}-g_{\beta}\right) \geqq \min \left\{h_{p}\left(g_{\alpha}+r_{\alpha, n} g\right), h_{p}\left(g_{\beta}+r_{\beta, n} g\right)\right\} \geqq n,
$$

for all positive integers $n$. Hence $h_{p}\left(g_{\alpha}-g_{\beta}\right)=\infty$.

PROOF OF THE THEOREM. Suppose $m>\aleph$. Then there is a set $S$ of $m$ independent elements, all having the same height $H$ and type $\tau$ maximal with respect to the property stated in the theorem. Let $g$ be one of these. By Lemma 1 the type of each $g_{\alpha} \in S$ different from $g$ increases in passing to $G /\{g\}_{*}$. It follows that there exists a subset $T$ of $S$ as described in Lemma 2 or Lemma 3. In either case the existence of such a set $T$ contradicts the maximality of the type $\tau$.

3. We conclude with the following characterizations of pure and $p$-pure subgroups of the additive group $P$ of $p$-adic integers.

Proposition 1. A torsion-free group $G$ is isomorphic to a pure subgroup of $P$ if and only if $G$ is homogeneous with the type of $P$ and is purely indecomposable.

Proposition 2. A torsion-free group $G$ is isomorphic to a p-pure subgroup of $P$ if and only if $G$ is p-reduced and p-purely indecomposable; i.e., $G$ has no nonzero subgroup $H$ such that $p H=H$ and every $p$-pure subgroup of $G$ is indecomposable. 
It is convenient to utilize the notion of a $p$-basic subgroup of a torsion-free group as presented in [3]. We refer the reader to this paper for definitions and properties of $p$-basic subgroups.

LEMмA. $A$ torsion-free group $G$ is p-reduced and has a cyclic p-basic subgroup if and only if $G$ is isomorphic to a p-pure subgroup of $P$.

Proof. Let $\{x\}$ be a $p$-basic subgroup of $G$. Define $\phi^{\prime}:\{x\} \rightarrow P$ by $\phi^{\prime}(x)=\pi \in P, \pi \neq 0$. Then we obtain the exact sequence $0=\operatorname{Hom}(G /\{x\}, P) \rightarrow \operatorname{Hom}(G, P) \rightarrow \operatorname{Hom}(\{x\}, P) \rightarrow \operatorname{Ext}(G /\{x\}, P)=0$. The left-hand equality follows since $G /\{x\}$ is $p$-divisible, and the right-hand equality since $\{x\}$ is $p$-pure in $G$ (see $[1, \S 3]$ ). Consequently, $\phi^{\prime}$ has a unique extension to $\phi$ mapping $G$ into $P$. Since $\operatorname{Ker} \phi$ is pure in $G, \rho_{p}(\operatorname{Ker} \phi)+\rho_{p}(\operatorname{Im} \phi)=\rho_{p}(G)=1$, where $\rho_{p}$ denotes the cardinality of a maximal $p$-independent subset [3, Lemma 4]. Since $\operatorname{Im} \phi \neq 0, \rho_{p}(\operatorname{Ker} \phi)=0$ and, therefore, $\phi$ is an isomorphism of $G$ into $P$. Moreover, $\operatorname{Hom}(G, P) \cong P$ which implies that $G$ is isomorphic to a $p$-pure subgroup of $P[1]$. Conversely, if $G$ is a $p$-pure subgroup of $P$, then $G$ contains a $p$-adic unit $u$ and $G /\{u\}$ is $p$-pure in

$$
P /\{u\} \cong \sum Q+\sum_{q \neq p} C\left(q^{\infty}\right) \text {. }
$$

Hence $G /\{u\}$ is $p$-divisible and since $\{u\}$ is $p$-pure in $G,\{u\}$ is a $p$-basic subgroup of $G$.

Proof of Proposition 1. The necessity is well known [2, Theorem 43.1]. Conversely, if $g \in G$ is an element of zero $p$-height, then since, by Lemma 1 , each element of $G /\{g\}_{*}$ has type greater than the type of $P$, it follows that $G /\{g\}_{*}$ is divisible. Hence $\{g\}$ is $p$-basic in $G$ and $G$ is isomorphic to a $p$-pure subgroup of $P$, which is pure since $G$ has the type of $P$.

Proof of Proposition 2. If $G$ is $p$-reduced and $p$-purely indecomposable, then $G$ has a cyclic $p$-basic subgroup and, by the lemma, $G$ is isomorphic to a $p$-pure subgroup of $P$. The converse was proven in $[1, \S 3]$.

\section{REFERENCES}

1. J. W. Armstrong, On p-pure subgroups of the p-adic integers, Topics in Abelian groups, Scott, Foresman and Company, Chicago, Ill., 1963; pp. 315-321.

2. L. Fuchs, Abelian groups, Hungarian Academy of Sciences, Budapest, 1958.

3. - Notes on abelian groups. II, Acta. Math. Acad. Sci. Hungar. 11 (1960), 117-125. 199.

4. E. Weinberg, Free lattice-ordered abelian groups, Math. Ann. 151 (1963), 187-

UNIVERSITY OF ILLINOIS 